\title{
Erratum: Dallago et al. Keeping Dairy Cows for Longer: A Critical Literature Review on Dairy Cow Longevity in High Milk-Producing Countries. Animals 2021, 11, 808
}

\author{
Gabriel M. Dallago $\left.{ }^{1} * \mathbb{(}\right)$, Kevin M. Wade ${ }^{1}\left(\mathbb{D}\right.$, Roger I. Cue ${ }^{1}$, J T. McClure ${ }^{2}$, René Lacroix ${ }^{3}$, Doris Pellerin ${ }^{4}$ \\ and Elsa Vasseur ${ }^{1}$ \\ 1 Department of Animal Science, McGill University, Sainte-Anne-de-Bellevue, QC H9X 3V9, Canada; \\ kevin.wade@mcgill.ca (K.M.W.); roger.cue@mcgill.ca (R.I.C.); elsa.vasseur@mcgill.ca (E.V.) \\ 2 Department of Health Management, Atlantic Veterinary College, University of Prince Edward Island, \\ Charlottetown, PE C1A 4P3, Canada; jmcclure@upei.ca \\ 3 Lactanet, Valacta, 555 Boul des Anciens-Combattants, Sainte-Anne-de-Bellevue, QC H9X 3R4, Canada; \\ rlacroix@lactanet.ca \\ 4 Département des Sciences Animales, Université Laval, Québec, QC G1V 0A6, Canada; \\ doris.pellerin@fsaa.ulaval.ca \\ * Correspondence: gabriel.dallago@mail.mcgill.ca
}

check for

updates

Citation: Dallago, G.M.; Wade, K.M.; Cue, R.I.; McClure, J.T.; Lacroix, R.; Pellerin, D.; Vasseur, E. Erratum: Dallago et al. Keeping Dairy Cows for Longer: A Critical Literature Review on Dairy Cow Longevity in High Milk-Producing Countries. Animals 2021, 11, 808. Animals 2021, 11, 2958. https://doi.org/ 10.3390/ani11102958

Received: 16 July 2021

Accepted: 26 September 2021

Published: 14 October 2021

Publisher's Note: MDPI stays neutral with regard to jurisdictional claims in published maps and institutional affiliations.

Copyright: (c) 2021 by the authors. Licensee MDPI, Basel, Switzerland. This article is an open access article distributed under the terms and conditions of the Creative Commons Attribution (CC BY) license (https:// creativecommons.org/licenses/by/ $4.0 /)$.
In the original article [1] there was a mistake in the published version of Tables 2 and 3 information about The Netherlands was missing in both Tables 2 and 3. The corrected Tables 2 and 3 appear below. The authors apologize for any inconvenience caused and state that the scientific conclusions are unaffected. The original article has been updated.

Table 2. The linear trend of milk yield $(\mathrm{kg})$ per animal per year for each country between 1961 and 2018. The list of countries is limited to the world's top high milk-producing countries for which we were able to provide sufficient and reliable data on the length of productive life.

\begin{tabular}{|c|c|c|c|c|c|}
\hline \multirow{2}{*}{ Country } & \multicolumn{2}{|c|}{ Model $^{1}$} & \multirow{2}{*}{$\mathbf{R}^{23}$} & \multirow{2}{*}{$\operatorname{RSE}^{4}$} & \multirow{2}{*}{$p$-Value ${ }^{5}$} \\
\hline & Intercept $^{2}$ & Year $^{2}$ & & & \\
\hline $\begin{array}{c}\text { United States of } \\
\text { America }\end{array}$ & $\begin{array}{c}2941.6^{* * *} \\
(40.6)\end{array}$ & $\begin{array}{c}129.7^{* * * *} \\
(1.20)\end{array}$ & 0.99 & 152.5 & $<0.001$ \\
\hline Brazil & $\begin{array}{c}451.2^{* * *} \\
(50.4)\end{array}$ & $\begin{array}{c}18.5^{* * *} \\
(1.49)\end{array}$ & 0.73 & 189.5 & $<0.001$ \\
\hline Germany & $\begin{array}{c}2904.6^{* * *} \\
(83.4)\end{array}$ & $\begin{array}{l}81.4^{* * *} \\
(2.46)\end{array}$ & 0.95 & 313.4 & $<0.001$ \\
\hline France & $\begin{array}{c}2103.8^{* * *} \\
(56.8)\end{array}$ & $\begin{array}{l}91.9^{* * *} \\
(1.68)\end{array}$ & 0.98 & 213.6 & $<0.001$ \\
\hline New Zealand & $\begin{array}{c}2419.1^{* * *} \\
(70.9)\end{array}$ & $\begin{array}{l}29.0^{* * *} \\
(2.09)\end{array}$ & 0.77 & 266.7 & $<0.001$ \\
\hline The Netherlands & $\begin{array}{c}3485.8^{* * *} \\
(61.2)\end{array}$ & $\begin{array}{l}84.3^{* * *} \\
(1.80)\end{array}$ & 0.97 & 230.0 & $<0.001$ \\
\hline Poland & $\begin{array}{c}1603.6^{* * *} \\
(107.4)\end{array}$ & $\begin{array}{l}65.3^{* * *} \\
(3.17)\end{array}$ & 0.88 & 403.8 & $<0.001$ \\
\hline Italy & $\begin{array}{c}2200.5^{* * *} \\
(91.3)\end{array}$ & $\begin{array}{l}72.5^{* * *} \\
(2.69)\end{array}$ & 0.93 & 343.1 & $<0.001$ \\
\hline Canada & $\begin{array}{c}2081.3^{* * *} \\
(84.1)\end{array}$ & $\begin{array}{c}120.7^{* * * *} \\
(2.48)\end{array}$ & 0.98 & 316.3 & $<0.001$ \\
\hline Ireland & $\begin{array}{c}2035.0 * * * \\
(53.3)\end{array}$ & $\begin{array}{l}59.9^{* * *} \\
(1.57)\end{array}$ & 0.96 & 200.5 & $<0.001$ \\
\hline
\end{tabular}

$1 * * *=p$-value $<0.01 ;{ }^{2}$ Estimate (standard error) $;{ }^{3} \mathrm{R}^{2}=$ Coefficient of determination; ${ }^{4} \mathrm{RSE}=$ Residual standard error; ${ }^{5}$ Model significance. 
Table 3. The linear trend of the length of productive life (year) in each country. The list of countries is limited to the world's top high milk-producing countries for which we were able to provide sufficient and reliable data on the length of productive life.

\begin{tabular}{|c|c|c|c|c|c|c|}
\hline \multirow{2}{*}{ Country } & \multirow{2}{*}{ Year } & \multicolumn{2}{|c|}{ Model $^{1}$} & \multirow{2}{*}{$\mathbf{R}^{23}$} & \multirow{2}{*}{$\operatorname{RSE}^{4}$} & \multirow{2}{*}{$p$-Value ${ }^{5}$} \\
\hline & & Intercept $^{2}$ & Year ${ }^{2}$ & & & \\
\hline United States of America & 1980-2019 & $\begin{array}{c}3.25^{* * *} \\
(0.10)\end{array}$ & $\begin{array}{c}0.0004^{\mathrm{NS}} \\
(0.004)\end{array}$ & 0.0003 & 0.30 & 0.92 \\
\hline Brazil & 1997-2018 & $\begin{array}{c}4.06^{* * *} \\
(0.24)\end{array}$ & $\begin{array}{c}-0.12^{* * *} \\
(0.02)\end{array}$ & 0.67 & 0.55 & $<0.001$ \\
\hline Germany & 1993-2019 & $\begin{array}{c}3.11^{* * *} \\
(0.07)\end{array}$ & $\begin{array}{l}0.01^{\mathrm{NS}} \\
(0.004)\end{array}$ & 0.13 & 0.18 & 0.06 \\
\hline France & 1968-2019 & $\begin{array}{c}3.89^{* * *} \\
(0.10)\end{array}$ & $\begin{array}{c}-0.04^{* * *} \\
(0.003)\end{array}$ & 0.76 & 0.37 & $<0.001$ \\
\hline New Zealand & 1982-2019 & $\begin{array}{c}3.69 * * * \\
(0.19)\end{array}$ & $\begin{array}{c}0.05^{* * *} \\
(0.01)\end{array}$ & 0.48 & 0.56 & $<0.001$ \\
\hline The Netherlands & 1970-2019 & $\begin{array}{c}3.38^{* * *} \\
(0.17)\end{array}$ & $\begin{array}{c}-0.01 \mathrm{NS} \\
(0.01)\end{array}$ & 0.05 & 0.59 & 0.12 \\
\hline Poland & 2003-2019 & $\begin{array}{l}6.81^{* * *} \\
(0.25)\end{array}$ & $\begin{array}{c}-0.19 \text { *** } \\
(0.02)\end{array}$ & 0.79 & 0.50 & $<0.001$ \\
\hline Italy & 1970-2019 & $\begin{array}{l}4.26^{* * *} \\
(0.14)\end{array}$ & $\begin{array}{c}-0.02 * * * \\
(0.005)\end{array}$ & 0.22 & 0.49 & $<0.001$ \\
\hline Canada & 1967-2019 & $\begin{array}{c}3.38^{* * *} \\
(0.13)\end{array}$ & $\begin{array}{c}-0.03 \text { *** } \\
(0.004)\end{array}$ & 0.57 & 0.47 & $<0.001$ \\
\hline Ireland & 1974-2019 & $\begin{array}{c}4.28^{* * *} \\
(0.21)\end{array}$ & $\begin{array}{c}-0.02 * \\
(0.01)\end{array}$ & 0.14 & 0.70 & 0.01 \\
\hline
\end{tabular}

${ }^{1} \mathrm{NS}=$ Not significant, ${ }^{*}=p$-value $<0.10,{ }^{* * *}=p$-value $<0.01 ;{ }^{2}$ Estimate (standard error) ${ }^{3} \mathrm{R}^{2}=$ Coefficient of determination; ${ }^{4}$ RSE $=$ Residual standard error; ${ }^{5}$ Model significance.

The authors would like to apologize for any inconvenience caused. The change does not affect the scientific results. The manuscript will be updated, and the original will remain online on the article webpage.

\section{Reference}

1. Dallago, G.M.; Wade, K.M.; Cue, R.I.; McClure, J.T.; Lacroix, R.; Pellerin, D.; Vasseur, E. Keeping Dairy Cows for Longer: A Critical Literature Review on Dairy Cow Longevity in High Milk-Producing Countries. Animals 2021, 11, 808. [CrossRef] [PubMed] 